\title{
GitHub Sponsors mở ra cơ hội cho các nhà phát triển phần mềm mã nguồn mở
}

\author{
Hồ Mạnh Toàn \\ A.I. for Social Data Lab, SSHPA System
}

Hà Nội, 28-05-2019

GitHub hiện nay là nền tảng chia sẻ, quản lý mã nguồn mở cho các dự án phát triển phần mềm lớn nhất thế giới hiên nay với hơn 37 triệu người dùng và 57 triệu kho mã nguồn. Trong nghiên cứu học thuật, GitHub cũng được các nhà khoa học tận dụng để lưu trữ dự án phát triển phần mềm của mình. Điển hình là kho lưu trữ của dự án Event Horizon Telescope [1], hay phần mềm thống kê Bayesian-bayesvl-trên môi trường $R[2,3]$.

Là một địa chỉ chia sẻ mã nguồn mở, vấn đề kinh phí luôn luôn gây đau đầu cho các nhà phát triển trên GitHub. Vì vậy, GitHub Sponsors được GitHub triển khai mới đây là một tín hiệu đáng mừng cho các nhà phát triển phần mềm đang đóng góp cho mã nguồn mở thông qua sản xuất phần mềm, thiết kế, sản xuất tài liệu tham khảo, hay cả hướng dẫn [4].

Chương trình này hoàn toàn không yêu cầu bất kì khoản phí nào và đảm bảo $100 \%$ tài trợ sẽ được chuyển đến tay nhà phát triển. Để cảm ơn người dùng, GitHub Sponsors cũng sẽ hỗ trợ chi phí xử lí trong 12 tháng đầu của chương trình. GitHub Sponsors không gói gọn trong khu vực nào mà mời gọi các nhà phát triển đến từ mọi nơi trên thế giới. Hiện nay, do đang trong giai đoạn beta nên GitHub Sponsors vẫn đang giới hạn các nhà phát triển có thể tham gia, tuy nhiên, người dùng nào cũng có thể tài trợ các nhà phát triển được tham gia chương trình.

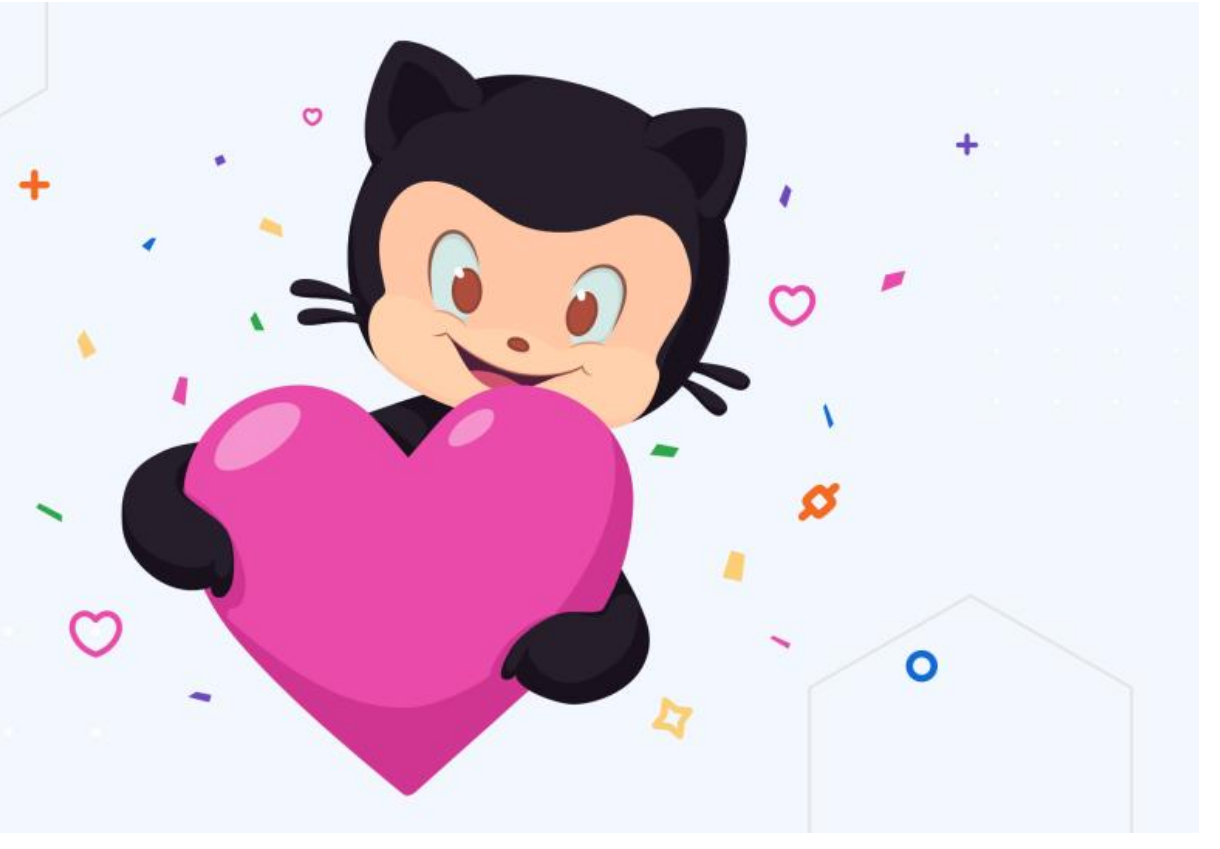


(Nguồn: The GitHub Blog)

Theo trang tin MakeUseOf [5], chương trình GitHub Sponsors chắc chắn sẽ khuyến khích các nhà phát triển đưa dự án của mình thành dự án mã nguồn mở. Tuy nhiên, việc tài trợ cộng đồng cũng có thể gây ra ảnh hưởng đáng kể tới dự án của nhà phát triển.

Đây cũng là một tín hiệu vui với khoa học khi công nghệ và xu hướng mở [6] đang thay đổi, giảm thiểu chi phí nghiên cứu học thuật [7]. Đồng thời, các nền tảng gọi vốn cộng đồng đang ngày một phổ biến hơn, góp phần tạo ra các cơ hội mới cho các nhà nghiên cứu, đặc biệt là những nhà nghiên cứu trẻ.

Note: Published in SciComm SSHPA: https://sc.sshpa.com/post/5481

\section{References:}

[1] Ho, T. M. (2019, April 15). Bức ảnh "mờ mịt": mỹ cảm khoa học, đức hy sinh và vận hội của một tạp chí. SciComm SSHPA. Retrieved from https://sc.sshpa.com/post/5449; DOI: $10.31219 /$ osf.io/g82kd.

[2] La, V.P, \& Vuong, Q.H. (2019). bayesvl: Visually Learning the Graphical Structure of Bayesian Networks and Performing MCMC with 'Stan'. The Comprehensive $R$ Archive Network (CRAN): <https://cran.rproject.org/web/packages/bayesvl/index.html>; version 0.8.5 (May 24, 2019).

[3] Vuong, Q. H., \& La, V. H. (2019). BayesVL package for Bayesian statistical analyses in R. Github: <https://github.com/sshpa/bayesvl>; V0.8.4.

[4] Zuegel, D. (201, May 23). Announcing GitHub Sponsors: a new way to contribute to open source. The GitHub Blog. Retrieved from https://github.blog/2019-05-23announcing-github-sponsors-a-new-way-to-contribute-to-open-source/ (May 28, 2019).

[5] Parrack, D. (2019, May 27). GitHub Sponsors lets you fund open source software. MakeUseOf. Retrieved from https://www.makeuseof.com/tag/github-sponsorsfund-open-source-software/ (May 28, 2019)

[6] Vuong, Q. H. (2017, December 12). Open data, open review and open dialogue in making social sciences plausible. Nature: Scientific Data Updates. Retrieved from: http://blogs.nature.com/scientificdata/2017/12/12/authors-corner-open-dataopen-review-and-open-dialogue-in-making-social-sciences-plausible/ (May 28, 2019).

[7] Vuong, Q. H. (2018). The (ir)rational consideration of the cost of science in transition economies. Nature Human Behaviour, 2(1), 5, DOI: https://doi.org/10.1038/s41562-017-0281-4. 